\author{
Military Technical College \\ Kobry Elkobbah, Cairo, \\ Egypt.
}

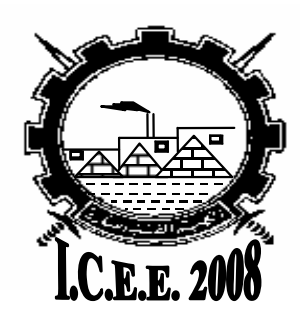

$4^{\text {th }}$ International Conference

On

Chemical \& Environmental

Engineering

27-29 May 2008

\title{
EFFECT OF GAMMA RADIATION ON THE ALBINO RAT'S LIVER AND THE POSSIBLE PROTECTIVE ROLE OF $\beta$-CAROTENE ON THE HISTOLOGICAL AND ULTRA STRUCTURAL CHANGES
}

\author{
M. I. Rady* , S. M. Abu Nour ${ }^{* *}$ and I. M. Khali|**
}

The present investigation reports the possible protective effect of $\beta$-carotene against hepatic lesions induced in male albino rats due to $\gamma$-irradiation. 48 male albino rats were divided into three main groups; the first group served as control (12 rats). Rats of the second group (18 rats) were exposed to a sub-lethal dose of whole body $y$ irradiation and then divided into three equal subgroups. The rats of the third main group (18 rats) received $5 \mathrm{mg} / \mathrm{kg}$ body wt of $\beta$-carotene at a daily oral dose for 2 consecutive weeks, and were then subjected to whole body $y$-irradiation at sub-lethal single dose level of 6Gy (Gray). This group was then divided into three equal subgroups. The rats were autopsied at three intervals; one week, two weeks and four weeks post irradiation. The obtained data revealed that $y$-irradiation-induced different histological changes in the liver of irradiated rats. These changes varied from haemorrhage, congestion in blood vessels, pyknosis and necrosis as well as complete degenerated areas in the liver. Electron micrographs recorded swollen mitochondria, fragmented endoplasmic reticulum, distorted nuclei and cell membrane and lipid infiltration in the hepatocytes of irradiated animals. The treatment with $\beta$ carotene $(5 \mathrm{mg} / \mathrm{kg}$ body wt for two consecutive weeks) pre-exposure to radiation attenuated most of these changes.

Therefore, the present study has concluded the potential use of $\beta$-carotene dissolved in corn oil as a radio protector.

\section{KEY WORDS:}

$\mathrm{Y}$-irradiation, albino rat, liver, $\beta$-carotene, histology, electron microscopy.

* Zoology Dept., Faculty of Science, Al Azhar University

** National Centre for Radiation Research and Technology (NCRRT), AEA. P. O. Box: 29 Nasr City, Egypt 


\section{INTRODUCTION}

The adverse effects of $y$-irradiation on the structure of different organs, including liver of experimental animals and human have been documented by many authors (Furuse et al., 2005 and Guryev, 2005).

lonizing radiation application to normal tissues results in fibrosis which is perhaps the most universal effect of radiation. In liver, radiation-induced late injury is histologically characterized by a loss of parenchymal cells (hepatocytes) and the distortion of the lobular architecture which is accompanied by both pericentral and periportal fibrosis (Geraci et al., 1992).

Electron microscopy of the liver of dogs exposed to chronic gamma-radiation demonstrated early changes in the hepatocyte structure at a relatively low dosage (Bespalova et al., 1979). Zavodnik et al. (2003) reported histological changes and alterations in biochemical parameters in liver of whole body gamma-irradiated rats. They added that gamma-radiation stimulated proliferation of the hepatocytes and induced time-dependent mitochondrial structural lesions of the hepatocytes; cytoplasmic vacuolization, and enhanced the number of lysosomes and of lipid inclusions. Radiotherapy is often used to treat patients with unresectable advanced hepatocellular carcinoma, where hypoalbuminemia, hyperbilirubinemia and ascites were important hepatic adverse events that develop after radiotherapy (Furuse et al., 2005).

Radio protective compounds are of importance in clinical radiation therapy, because normal tissues should be protected against radiation injury, when using higher doses of radiation (Nair et al., 2004). Vitamins are one of the several pharmacological compounds that could act as radio protectors or curative agents to damaging effects of radiation exposure (Kafafy, 2000). Nair et al. (2004) reported that, administration of tocopherol monoglucoside (water-soluble glycosylated derivative of vitamin E) immediately after exposure to gamma-radiation can protect normal tissues against radiation-induced damage in mice. In addition, Satyamitra et al. (2001) declared that the high water solubility and effectiveness of tocopherol monoglucoside favour its administration as a likely candidate for protection in case of accidental exposures. Thus, the present study was designed to investigate the histological and ultra structural changes in the liver induced by gamma-radiation of male rats. Also, an evaluation of the role of $\beta$-carotene as radio protective agent was carried out.

\section{MATERIALS AND METHODS}

The experimental animals were 48 male albino rats (Rattus norvegicus) with average body wt of $100-130 \mathrm{~g}$, and were purchased from the Egyptian Organization for Vaccine and Biological Preparations at Helwan. They were allowed two weeks preexperimentation period to be adapted to the laboratory conditions. Food and water were supplied daily ad libitum. Rats were subsequently divided into three main groups; the first one (12 rats) served as control. Rats of the second group (18 rats) were subjected to whole body $\mathrm{y}$-radiation at a sub-lethal single dose level of $6 \mathrm{~Gy}$ and then divided into three equal subgroups where the first subgroup was sacrificed after one week, the second after two and the third after four weeks of irradiation. Rats from the third main group (18 animals) received orally a dose of $5 \mathrm{mg} / \mathrm{kg}$ body wt. $\beta$ carotene daily for 2 consecutive weeks according to El-Habit et al. (2000) and were 
then subjected to whole body $Y$-rays at a sub-lethal single dose level of 6Gy. This group was then divided into three equal subgroups; the first subgroup was sacrificed after one week, the second after two and the third sacrificed after four weeks of exposure. Whole body $\mathrm{Y}$-irradiation was performed using ${ }^{137}$ Cesium Biological $\mathrm{Y}$-cell40 irradiation facility belonging to National Center for Radiation Research and Technology (NCRRT), Atomic Energy Authority (AEA). Whole body y-irradiation was delivered for the necessary calculated time for the sub-lethal dose level of $6 \mathrm{~Gy}$ (single dose). The dose rate was equal to $0.908 \mathrm{rad} / \mathrm{sec}$. The material used in the present investigation as a protective agent was $\beta$-carotene; this was manufactured by Fulka Co. Switzerland. It was diluted with corn oil at reasonably dilute concentration. The liver of control and treated animals were dissected out and washed in saline to remove the blood then cut into pieces and put in fixatives for routine histological evaluation, the tissues were fixed in 10\% buffered formalin (Lillie, 1954), dehydrated in an ascending series of ethyl alcohol and embedded in paraffin wax (melting point $\left.56{ }^{\circ} \mathrm{C}\right)$. Sections $(5-7 \mu$ thickness) were stained with haematoxylin and eosin and mounted in thin film of Canada balsam (Harris's, 1900). For electron microscopic examination tissue blocks were fixed in $4 \%$ glutaraldehyde in $0.2 \mathrm{M}$ sodium cacodylate buffer, post fixed in $2 \%$ osmium tetroxide in $0.3 \mathrm{M}$ sodium cacodylate, dehydrated in ethyl alcohol and embedded in an Araldite-Epon (EM Bed 812) Weakley (1981). Sections were examined using a transmission electron microscope (JEM 100X CX) at NCRRT.

\section{RESULTS}

\section{Histological Studies}

Liver sections of control rats showed the common characteristic lobular organization; each lobule is formed of radially arranged cords of hepatocytes that extend from the central vein. The hepatic cords are separated by narrow blood sinusoids lined with two types of cells, endothelial (with thin, small, rod-like nucleus) and von-Kupffer cells (containing oval dense nuclei). The hepatocytes are large spherical cells with spherical nuclei and prominent deeply stained nucleoli (Fig. 1).

Liver sections of rats' one week post $\mathrm{Y}$-irradiation (6Gy) revealed different histopathological alterations. These alterations were manifested as dilatation and congestion in blood vessels and appearance of inflammatory cells. The hepatocytes showed vacuolated cytoplasm and often with nuclei. Blood sinusoids were dilated (Fig. 2). Two weeks post gamma- irradiation liver sections exhibited an increase in the above mentioned effects. Blood vessels were congested, more dilated and infiltrated with inflammatory cells. Blood sinusoids showed also dilatation, and hepatocytes revealed pyknotic nuclei (Fig. 3). Moreover, necrotic hepatocytes were encountered in different areas of the liver tissue. Four weeks post $\mathrm{Y}$-irradiation; rat liver sections displayed more pronounced deleterious histological changes (Fig. 4 \& 5). Hepatocytes were completely degenerated and increased numbers of fibrocytes were found within the tissues. Furthermore, in necrotic areas, haemorrhage, obvious dilatation of blood vessels and blood sinusoids and invasion of both endothelial and Kupffer cells were noticed. The pre-treatment of rats with $\beta$-carotene for two weeks before exposure to $\gamma$-radiation attenuated the deleterious effects of radiation. Liver sections of these rats one week post irradiation revealed mostly normal hepatocytes 
with noticeable increase of endothelial and Kupffer cells within the tissues (Fig. 6). Yet, dilated blood vessels disrupted lining and dilated blood sinusoids were mostly still evident.

Two weeks post $y$-irradiation the liver of rats pre-treated with $\beta$-carotene exhibited improvement of the hepatocytes in different parts of the tissues while the lymphocytic infiltration and appearance of large number of macrophages were still detected (Fig. 7). Also, pyknosis and necrosis were observed and few fibrocytes were found in different parts of the hepatic tissue. Slightly protective effect of $\beta$-carotene administered before $\mathrm{Y}$-irradiation could be detected four weeks post irradiation. Many of the hepatocytes appeared normal and haemorrhage disappeared. Nevertheless, other hepatocytes were abnormal with either pyknotic or karyolytic nuclei (Fig. 8). Also, few of fibrocytes, slightly dilated blood vessels and dilated blood sinusoids were noticed.

\section{Ultra Structural Studies}

The ultra structure of a normal hepatocyte is presented in figure 9. Electron micrographs showed ultra structural changes in different hepatocyte's components after one, two and four week's of exposure to the single dose of 6Gy gammaradiation. Electron micrographs of the hepatocytes after four weeks of exposure to $y$ radiation revealed focal degeneration of cytoplasmic matrix accompanied by lipid droplets of variable sizes. Also, nuclear lesions were observed as ill-defined electron translucent nuclei with ruptured membrane and irregular outer boundary. Micro coagulation of chromatin material with its migration to the nuclear periphery and fenestration of the nucleoli were noticed (Fig. 10). In addition, the blood sinusoids exhibited prominent dilatation and cell membranes in some cells were ruptured (Fig. 10). Swollen mitochondria with rupture or loss of its cristae were mostly prevailing in the hepatocytes after one, two and four weeks of exposure. Also, fragmentation of the endoplasmic reticulum was commonly detected where those encountered suffered vesiculation and rupture of lamellae and also, Golgi bodies were undistinguishable and detached ribosomes, which were particularly accumulated around mitochondria, were a common feature as shown in ( Fig. 11). Ultra structurally, the treatment of rats with $\beta$-carotene for two weeks before exposure to $\gamma$ radiation minimized most of the injuries noticed in the hepatocytes of rats exposed to radiation only. Regular round nuclei, normal nuclear membrane and normal mitochondria were displayed in the hepatocytes of rats treated with $\beta$-carotene and then exposed to gamma-radiation. These improvements were noticed even four weeks post gamma-radiation exposure (Fig. 12). Also, the cell membrane of the hepatocytes probably exhibited normal appearance with developing desmosomes in most of the cells and these cells showed lesser numbers of lysosomes (Fig. 13). Regeneration in the rough endoplasmic reticulum and obvious decrease in the lipid droplets were the most prominent features of the hepatocytes (Fig. 14). Meanwhile, some swollen mitochondria and fragmented endoplasmic reticulum were still found in different hepatocytes (Fig.13 and 14). 


\section{RESULTS}

\section{Histological Studies}

Liver sections of control rats showed the common characteristic lobular organization; each lobule is formed of radially arranged cords of hepatocytes that extend from the central vein.

The hepatic cords are separated by narrow blood sinusoids lined with two types of cells, endothelial (with thin, small, rod-like nucleus) and von-Kupffer cells (containing oval dense nuclei).

The hepatocytes are large spherical cells with spherical nuclei and prominent deeply stained nucleoli (Fig. 1).

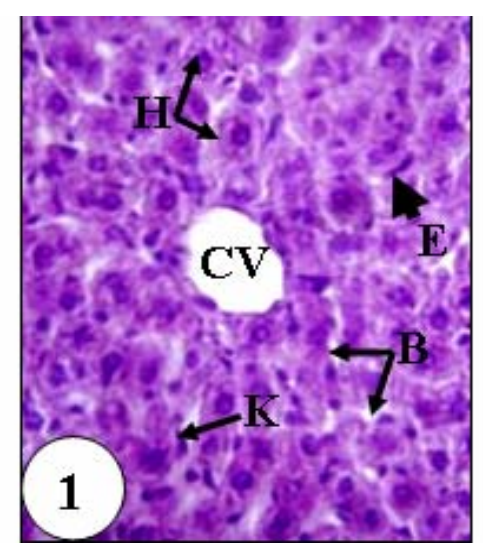

Fig. 1. Photomicrograph of control liver of an albino rat showing normal structure; hepatocytes $(\mathrm{H}$.$) , central vein (\mathrm{CV})$, blood sinusoid $(\mathrm{BS})$, endothelial cell $(\mathrm{E})$ and Kupffer cell (K). (H/E. X400).

Liver sections of rats' one week post Y-irradiation (6Gy) revealed different histopathological alterations. These alterations were manifested as dilatation and congestion in blood vessels and appearance of inflammatory cells.

The hepatocytes showed vacuolated cytoplasm and often with nuclei. Blood sinusoids were dilated (Fig. 2).

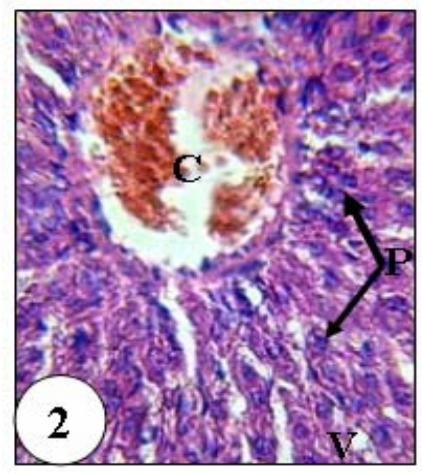

Fig. 2. Photomicrograph of liver of an albino rat irradiated with a single dose of $6 \mathrm{~Gy}$ after 1 week after the exposure showing congested and dilated blood vessel $(\mathrm{CB})$, pyknosis in the hepatocyte's nuclei $(\mathrm{P})$ and vacuolated hepatocyte's cytoplasm (V). (H/E. X 400). 
Two weeks post $y$-irradiation liver sections exhibited an increase in the abovementioned effects. Blood vessels were congested, more dilated and infiltrated with inflammatory cells. Blood sinusoids showed also dilatation, and hepatocytes revealed pyknotic nuclei (Fig. 3)

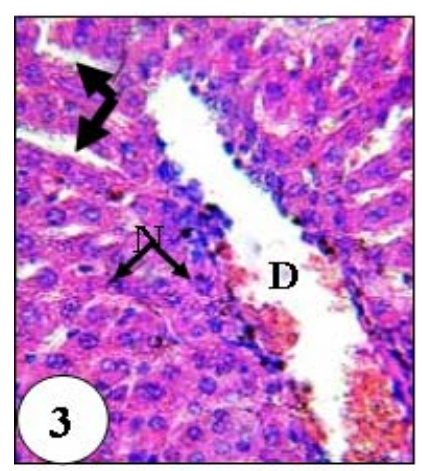

Fig. 3. Photomicrograph of liver of an albino rat irradiated with a single dose of $6 G y$ after 2 weeks from the exposure showing dilated blood sinusoids (thick arrows), dilated blood vessel (DB) and necrosis (N). (H/E. X 400).

Moreover, necrotic hepatocytes were encountered in different areas of the liver tissue. Four weeks post $\mathrm{Y}$-irradiation; rat liver sections displayed more pronounced deleterious histological changes (Fig. $4 \& 5$ ).
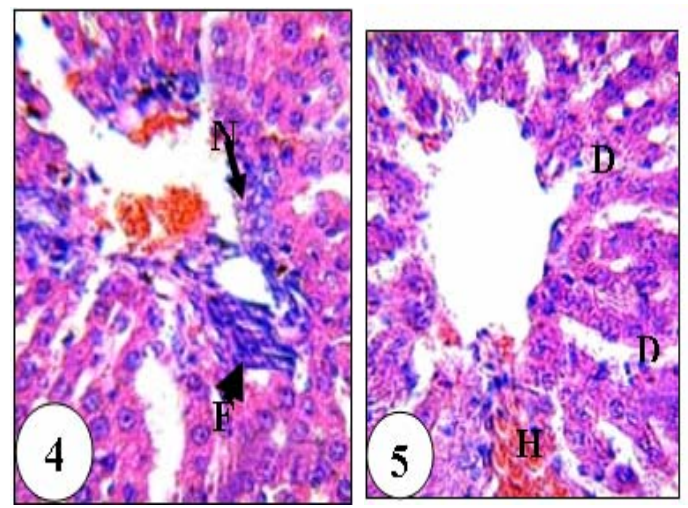

Fig. 4. Photomicrograph of liver of an albino rat irradiated with a single dose of $6 \mathrm{~Gy}$ after 4 weeks of exposure showing necrotic area (NA) and increase of fibrocytes (F). (H/E. X 400).

Fig. 5. Photomicrograph of liver of an albino rat irradiated with a single dose of $6 \mathrm{~Gy}$ after 4 weeks of exposure showing degeneration in hepatic tissue (D) and haemorrhage $(H)$. (H/E. X 400). 
Hepatocytes were completely degenerated and increased numbers of fibrocytes were found within the tissues. Furthermore, in necrotic areas, haemorrhage, obvious dilatation of blood vessels, blood sinusoids and invasion of both endothelial and Kupffer cells were noticed. The pre-treatment of rats with $\beta$-carotene for two weeks before exposure to gamma-radiation attenuated the deleterious effects of radiation. Liver sections of these rats one week post irradiation revealed mostly normal hepatocytes with noticeable increase of endothelial and Kupffer cells within the tissues (Fig. 6)

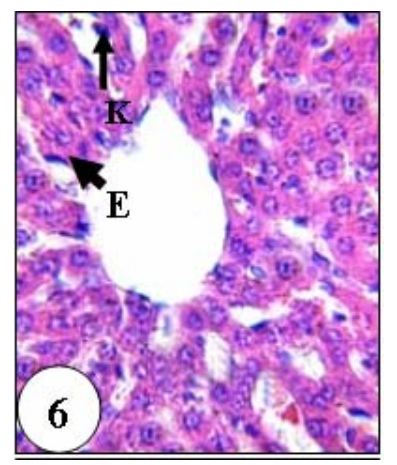

Fig. 6. Photomicrograph of liver of an albino rat pre-treated with Beta-carotene for 2 weeks, then irradiation with single dose of 6Gy after 1 week of the radiation exposure showing normal arrangement of the hepatic cords with invasion of endothelial cells (E) and Kupffer (K) cells. (H/E. X 400).

Yet, dilated blood vessels disrupted lining and dilated blood sinusoids were mostly still evident. Two weeks post $\gamma$-irradiation the liver of rats pre-treated with $\beta$-carotene exhibited improvement of the hepatocytes in different parts of the tissues while the lymphocytic infiltration and appearance of large number of macrophages were still detected (Fig. 7\&8).

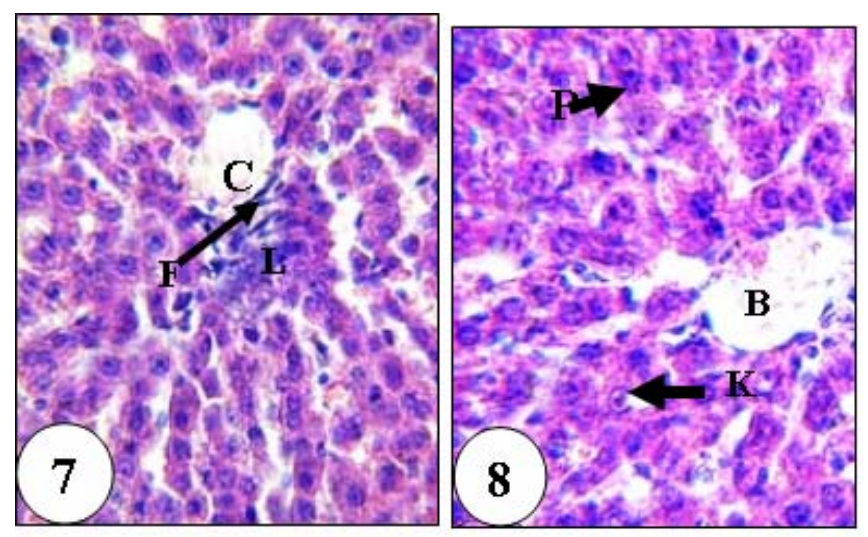

Fig. 7. Photomicrograph of liver of an albino rat pre-treated with Beta-carotene for 2 weeks, then irradiation with single dose of $6 \mathrm{~Gy}$ after 2 weeks of the exposure, showing regeneration in the hepatic cells with normal central vein (CV), lymphocyte infiltration (L) and less fibrocytes (F). (H/E. X 400).

Fig. 8. Photomicrograph of liver of an albino rat pre-treated with Beta-carotene for 2 weeks, then irradiation with single dose of $6 \mathrm{~Gy}$ after 4 weeks of the radiation exposure, showing karyolysis (Ka) and pyknosis $(\mathrm{P})$ with thin walled blood vessel (BV). (H/E. X 400). 
Also, pyknosis and necrosis were observed and few fibrocytes were found in different parts of the hepatic tissue. Slightly protective effect of $\beta$-carotene administered before $\gamma$-irradiation could be detected four weeks post irradiation.

Many of the hepatocytes appeared normal and haemorrhage disappeared. Nevertheless, other hepatocytes were abnormal with either pyknotic or karyolytic nuclei, or few of fibrocytes, slightly dilated blood vessels and dilated blood sinusoids were noticed (Fig.8)

\section{Ultra Structural Studies}

The ultra structural of a normal hepatocyte is presented in (Fig. 9).

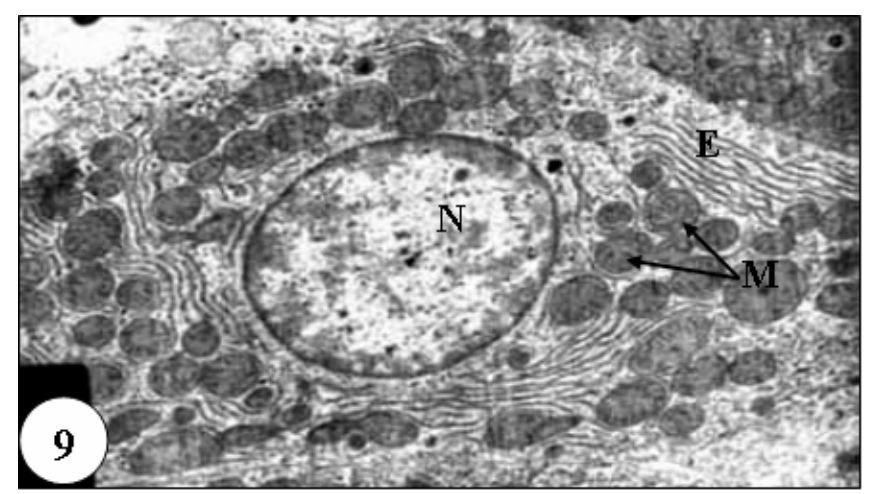

Fig. 9. Electron micrograph of control rat liver cell showing normal nucleus $(N)$, normal mitochondria (M) and normal endoplasmic reticulum (ER). (X 4000).

Electron micrographs showed ultra structural changes in different hepatocyte components after one, two and four week's of exposure to the single dose of $6 \mathrm{~Gy}$ gamma-radiation. Electron micrographs of the hepatocytes after four weeks of exposure to gamma-radiation revealed focal degeneration of cytoplasmic matrix accompanied by lipid droplets of variable sizes. Also, nuclear lesions were observed as ill-defined electron translucent nuclei with ruptured membrane and irregular outer boundary. Microcoagulation of chromatin material with its migration to the nuclear periphery and fenestration of the nucleoli were noticed (Fig. 10). 


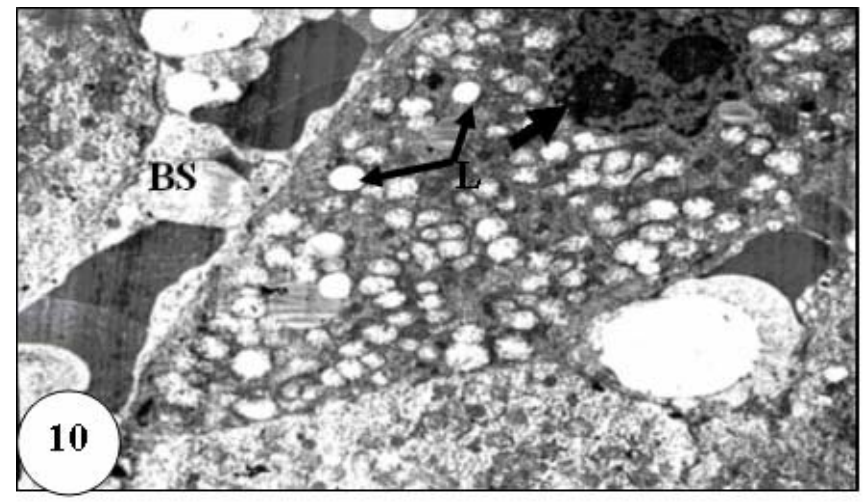

Fig. 10. Electron micrograph of a section of hepatocyte of an albino rat irradiated with single dose of 6Gy after 4 weeks from the exposure showing severe degeneration in the hepatocyte with electron dense nuclear chromatin and irregularity in nuclear membrane (arrow), notice: lipid droplets (L) and dilated blood sinusoids (BS). (X 3000).

In addition, the blood sinusoids exhibited prominent dilatation and cell membranes in some cells were ruptured (Fig. 10). Swollen mitochondria with rupture or loss of its cristae were mostly prevailing in the hepatocytes after one, two and four weeks of exposure.

Also, fragmentation of the endoplasmic reticulum was commonly detected where those encountered suffered vesiculation, rupture of lamellae, also Golgi bodies were undistinguishable and detached ribosomes, which were particularly accumulated around mitochondria, as a common feature as shown in (Fig. 11) .

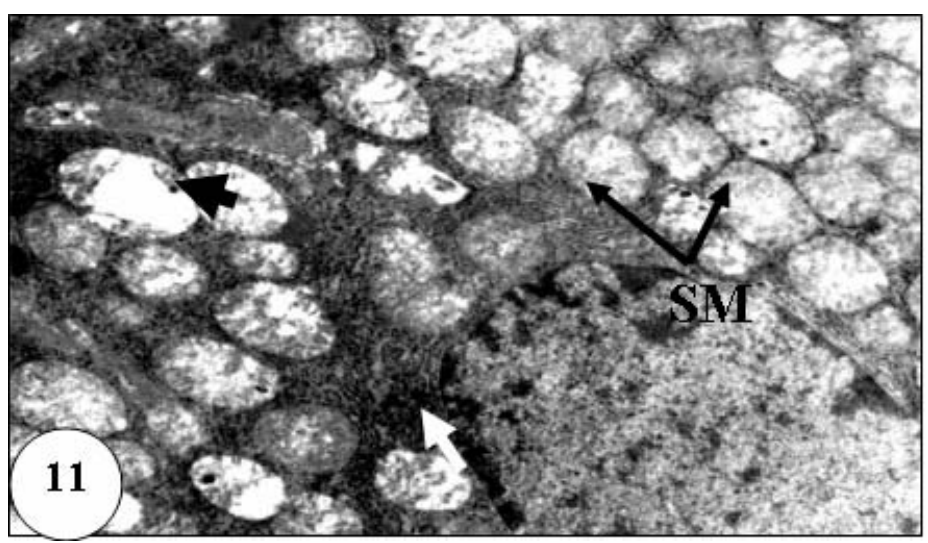

Fig. 11. Electron micrograph of a section of hepatocyte of an albino rat irradiated with single dose of $6 \mathrm{~Gy}$ after 4 weeks from the exposure showing swollen mitochondria (SM), rupture in its cristae (thick arrow) and fragmentation of endoplasmic reticulum (white arrow). (X 6000).

Ultrastructurally, the treatment of rats with $\beta$-carotene for two weeks before exposure to $\mathrm{y}$-radiation minimized most of the injuries noticed in the hepatocytes of rats exposed only to radiation. Regular round nuclei, normal nuclear membrane and 
normal mitochondria were displayed in the hepatocytes of rats treated with $\beta$ carotene and then exposed to gamma-radiation. These improvements were noticed even four weeks post gamma-radiation exposure (Fig. 12).

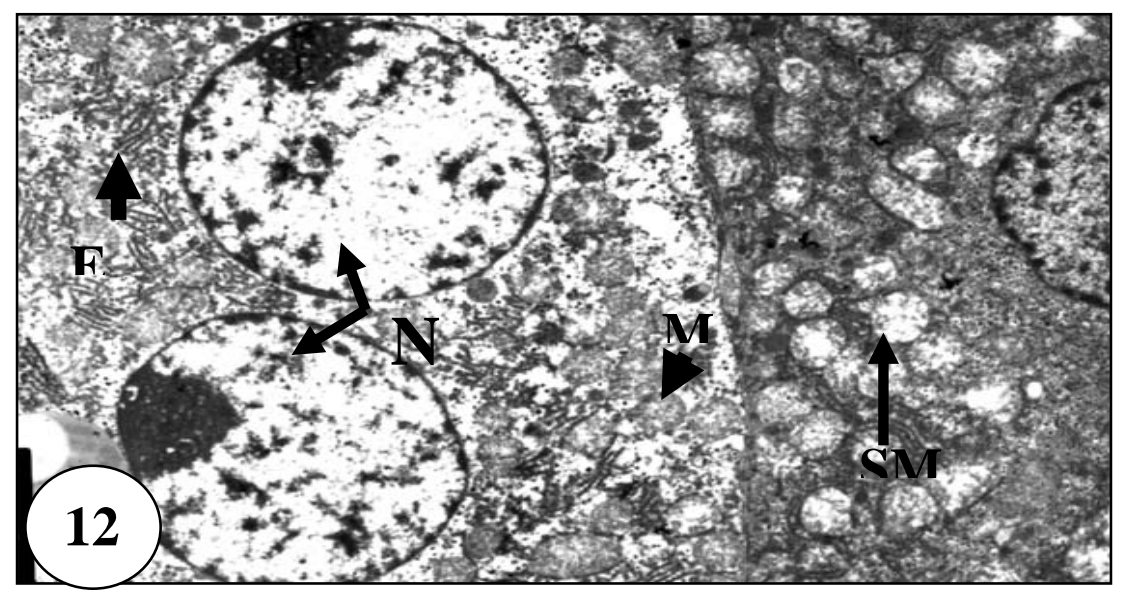

Fig. 12. Electron micrograph of hepatocyte section of an albino rat after treatment with beta-carotene for 2 weeks, then irradiation with single dose of $6 \mathrm{~Gy}$ after 4 weeks from the radiation exposure showing regeneration in the hepatocyte, notice: binucleated $(\mathrm{N})$ cells, improvement of mitochondria $(\mathrm{M})$, endoplasmic reticulum $(E R)$ and intact cell membrane between 2 hepatocytes. The other right cell shows some swollen mitochondria (SM). (X 6000).

Also, the cell membrane of the hepatocytes probably exhibited normal appearance with developing desmosomes in most of the cells and these cells showed lesser numbers of lysosomes (Fig. 13).

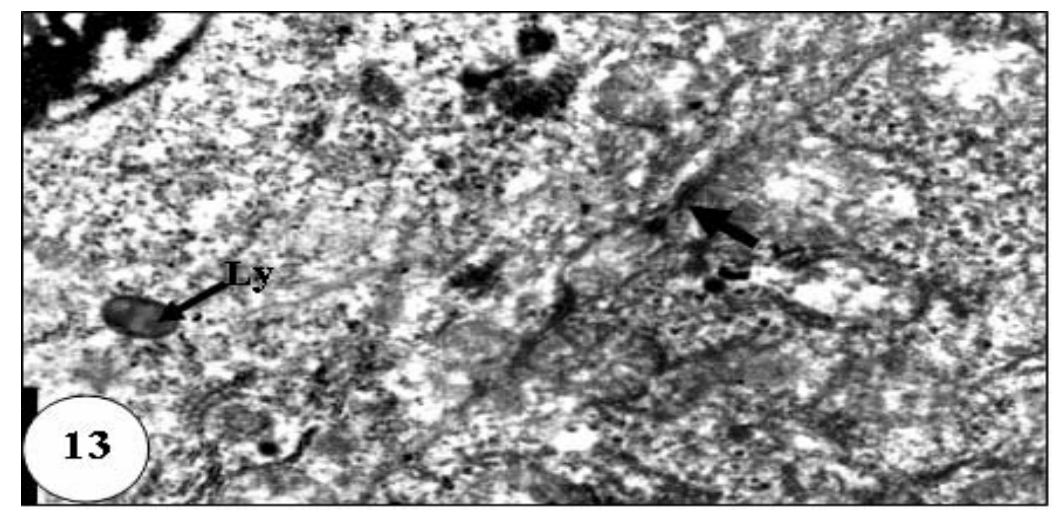

Fig. 13. Electron micrograph of hepatocyte section of an albino rat after treatment with beta-carotene for 2 weeks, then irradiation with single dose of 6Gy after 4 weeks from the radiation exposure, showing an intact cell membrane between 2 hepatocytes, decrease of lysosomes (Ly) and desmosome can be seen (thick arrow). (X 6000).

Regeneration in the rough endoplasmic reticulum and obvious decrease in the lipid droplets were the most prominent features of the hepatocytes (Fig. 14). 


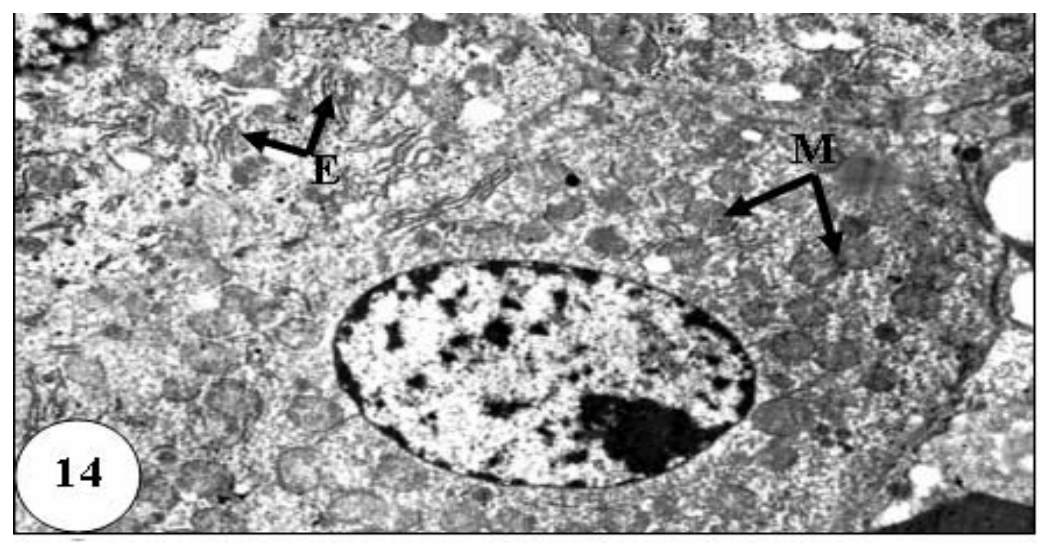

Fig. 14. Electron micrograph of hepatocyte section of an albino rat after treatment with beta-carotene for 2 weeks, then irradiation with single dose of 6Gy after 4 weeks from the radiation exposure, showing improvement in the hepatocytes; normal mitochondria $(\mathrm{M})$ and regeneration in the endoplasmic reticulum (E). Round shape nucleus with regular nuclear membrane were found. (X 6000).

Meanwhile, some swollen mitochondria and fragmented endoplasmic reticulum were still found in different hepatocytes (Figs.13 and 14).

\section{DISCUSSION}

The present results revealed that, the liver of gamma-irradiated rats exhibited varying lesions that included congestion, dilatation in blood vessels, haemorrhage, vacuolation and necrobiotic changes in the hepatocytes. In addition, the increase in lymphocyte infiltrations and fibrocytes were demonstrated. Similar results were documented by many authors on different experimental animals exposed to gammaradiation (Yarmonenko, 1998, Zavodnik et al., 2003 and Guryev, 2005). Degeneration and necrosis of the hepatic cells following exposure to radiation could be explained according to the suggestion of Ritter (1977) who reported that liver cell necrosis may be either due to progressive degenerative action of intracellular enzymes of the injured cells or to a metabolic disturbance and inhibition of synthesis needed of DNA and hence protein synthesis for the growth and maturation of the liver. It is well established that most of the physiological activities in the animal body are disturbed after exposure to ionizing radiation. These disturbances are either due to the direct harmful effects of irradiation on the biological systems or indirect effect of free radicals liberated in the body after irradiation (Hagen, 1989). Also, Geraci and Mariano (1993) suggested that radiation-induced alterations of a nonparenchymal cell population in liver might have been responsible for fibrosis which led to hepatocytes loss. Exposure to doses of ionizing radiation is associated with physiopathological changes. These changes differ in their severity according to the radiosensitivity and responses of individual organs and tissues (Yarmonenko, 1998). The present findings indicated that the effects of gamma radiation on the liver of male rats increased at the fourth week post exposure than after one or two weeks. In coincidence, Geraci et al. (1992) reported that, it is frequently a dose-limiting 
complication; this late complication has been viewed as the delayed expression of radiation injury, which follows several weeks to months after radiation. Electron microscopy showed that whole body irradiation induced different ultrasturctural changes in the hepatocytes of treated rats. These changes were represented as swollen and ruptured mitochondria, fragmentation of the endoplasmic reticulum, nuclear damage and lipid infiltration in the cytoplasm. The aforesaid alterations in the liver tissue showed similarity and conforms those recorded by several authors, with various experimental animals exposed to radiation (Nasr et al., 1993, Zavodnik et al., 2003 and Hussein et al., 2005). Swollen or hydropic mitochondria delineated in the concurrent study may be due to the entry of water which can be engendered by agent that produce cell damage (Ghadially, 1983). Fragmentation or vesiculation of the endoplasmic reticulum observed in the present work is due to ingress of water and solutes into the cell due to dysfunction of the cell membrane permeability that may lead to dilatation and vesiculation of the endoplasmic reticulum (Nasr et al., 1993). The cytoplasmic vacuolation observed in the liver cells as a result of exposure to radiation could be explained partly by inhibition of fat mobilization from the liver as discussed by Schenker (1975). Other authors are of the opinion that cytoplasmic vacuolation is most probably brought about by the increase of lysosome elements (El-Banhway et al., 1993). Ongoing trials for protection from gamma radiation effects by using chemicals or other means are still of interest to many radiobiologists, to achieve less toxic substances with maximum protecting action against radiation effects (Eissa and Moustafa, 2001). Vitamins are one of the several pharmacological compounds that could act as radio protectors or curative to damaging effect of radiation exposure (Kawaski and Sakarai, 1969 and Kafafy, 2000). The present results showed that the treatment of rats with $\beta$-carotene dissolved in corn oil for two weeks prior to gamma irradiation could attenuate the adverse effects of radiation exposure. Most of the histopathological lesions observed after gamma-irradiation disappeared to a large extent; where the normal architecture of the liver was restored, whereas, few fibrocytes, slight dilated blood vessels and blood sinusoids were still found. Also, similar results were obtained with electron microscopy. The mitochondria, endoplasmic reticulum, cell membrane and nuclei were mostly normal. However, Zavodnik et al. (2003) concluded that the whole body single gammairradiation with a dose of $1 \mathrm{~Gy}$ led to reversible but significant damages to the rat's liver cell membrane structure and these damages might be the reason of radiationinduced liver morphological alterations. Also, electron microscopy revealed different binucleated hepatocytes in animals treated with $\beta$-carotene for two weeks and then exposed to $\gamma$-radiation. Guryev (2005), suggested that a high number of binucleated hepatocytes could be produced as a result of the cell dividing damage (acytokinesis) that could lead to adaptive response to low-dose rate radiation. Similarly, Korolev et al. (1996) showed that an intake course of sulphate mineral water after total radiation (1 and 5 Gy) can prevent and minimize dystrophic and necrobiotic changes in the liver, stimulate intracellular regeneration and reduce pathological mitosis. Depletion in endogenous antioxidants mainly glutathione occur due to oxygen radicals generated by gamma-irradiation (Bhatia and Jain, 2004) and hence proteins located within membranes may become more susceptible to enzymatic degradation following 
oxidative damage (Grant et al., 1993). It is possible to conclude that beta-carotene dissolved in corn oil could induce a protective role against radiation-induced liver lesions. This action could be attributed to its antioxidant effect.

\section{RECOMMENDATIONS}

- Avoiding gamma-radiation as much as possible.

- Using all the precaution means when dealing with gamma-radiation.

- Using beta-carotene in diet specially for people who works in the radiation field or under radiotherapy.

- Regular check up on people who expose to gamma-radiations in their work.

\section{REFERENCES}

[1] Bespalova, L. A., Shikhodyrov, V. V. and Romanov, V. S. (1979) Ultrastructural changes in the hepatocytes of dogs continuously exposed to chronic low-dose gamma irradiation. Kosm. Biol. Aviakosm. Med., 13(5): 65.

[2] Bhatia, A. L. and Jain, M. (2004) Spinacia oleracea L. protects against gamma radiations: as a study on glutathione and lipid peroxidation in mouse liver. Phytomed., 11(7-8): 607.

[3] Eissa, O. and Moustafa, A. (2001) Histochemical studies on the role of pantothenic acid on radiation damage of the kidney. Egypt. J. Hosp. Med., 2, 163.

[4] El-Banhawy, M. A. Sanad, S. M., Zahaby, A. S. and Eid, R. M. (1993) An electron microscopic investigation on the effect of one of the environmental pollutants "dursban insecticide "on the mammalian liver. J. Egypt. Ger. Soc. Zool., 12(C): 287.

[5] El-Habit, O. H. M., Saada, H. N., Azab, K. S., Abdel-Rahman, M. and ElMalah, D. F. (2000) The modifying effect of $\beta$-carotene on gamma radiationinduced elevation of oxidative reactions and genotoxicity in male rats. Mut. Res. Gene. Toxicol. Environ. Muatgen., 466(2): 179.

[6] Furuse, J. Ishii, M., Nagase, M., Kawashima, M., Ogino, I. and Yoshino, M. (2005) Adverse hepatic events caused by radiotherapy for hepatocellular carcinoma. J. Gastroenterol. Hepatol., 20(10): 1512.

[7] Geraci, J. P. Mariano, M. S. and Jackson, K. L. (1992) Radiation hepatology of the rat: Microvascular fibrosis and enhancement of liver dysfunction by diet and drugs. Rad. Res., 129, 322.

[8] Geraci, J. P. and Mariano, M. S. (1993) Radiation hepatology of the rat: Parenchymal and nonparynchymal cell injury. Rad. Res., 136, 205.

[9] Ghadially, N. F. (1983) Ultrastructural pathology of cell and matrix structure. $3^{\text {ed }}$ ed. London, Boston, Sydney.

[10] Grant, A. J., Jessup, W. and Dean, R. T. (1993) Enhanced enzymatic degradation of radical damaged mitochondrial membrane components. Free Rad. Res. Commun., 19(2): 125.

[11] Guryev, D. V. (2005) Histologic assessment of regenerating rat liver under lowdose rate radiation exposure. Inter. Congress Series, 1276, 181. 
[12] Hagen, V. (1989) Biochemical aspects of radiation biology. Experientia, 45, 7.

[13] Harris's, H.F. (1900): Histochemical Techn. By Methuen and Co. Ltd.

[14] Hussein, M. R., Abu-Dief, E. E., Abd el-Reheem, M. H. and Abd-Elrahman, A. (2005) Ultrastructural evaluation of the radioprotective effects of melatonin against X-ray-induced skin damage in Albino rats. Inter. J. Experim. Pathol., 86(1): 45.

[15] Kafafy, Y. A. (2000) Protective effect of cystine and vitamin E on gamma irradiation injury in rats. Egyp. J. Rad. Sci. Applic., 13, 2.

[16] Kawasaki, S. and Sakarai, K. (1969) Effect of some drugs on recovery from radiation damage. Nippon Igaku Hoshasen Gakkai Zasshi, 28, 1375.

[17] Korolev, I., Panova, L. N., Nikulina, L. A. and Zagorskaia, N. Z. (1996) The action of sulfate mineral water in experimental whole-body irradiation. Vopr. Kurortol. Fizioter. Lech. Fiz. Kult, 1, 25.

[18] Lillie, R.D. (1954): Histopathological techniques and practical istochemistry. Mc Grow-Hill, U.S.A.

[19] Nair, C. K., Salvi, V., Kagiya, T. V. and Rajagopalan, R. (2004) Relevance of radioprotectors in radiotherapy: studies with tocopherol monoglucoside. J. Environ. Pathol. Toxicol. Oncol., 23(2): 153.

[20] Nasr, S. A., Wahba, S. R., Roushdy, H. M. and Abou-Nour, S. A. (1993) Misonidazole sensitization of radiation-induced ultrastructural alterations in the liver of rat. J. Egypt. Ger. Soc. Zool., 10(C): 29.

[21] Ritter, E. J. (1977) Altered Biosynthesis. In: Handbook of Teratology. Vol.2 Plenum Press, New York.

[22] Satyamitra, M., Devi, H., Murase, H. and Kagiya, V. T. (2001) In vivo radioprotection by alpha-TMG: preliminary studies. Mut. Res., 479(1-2): 53.

[23] Schenker, S. (1975) Drug and the liver. W. Gerok and K.sickinger (eds), Stuttgar.F. K.Schattauer. Veralge. p. 269.

[24] Weakley, B.S. (1981): A beginner's Handbook in biological transmission electron microscopy, 2nded., London, Churchill Livingstone.

[25] Yarmonenko, S. P. (1998) In radiobiology of human and animals. Mir. Publisher, Moscow, p. 33.

[26] Zavodnik, L. B., Kravchuk, R., Artsukevich, A., Chumachenko, S., Sheibak, M., Ovchinnikov, V. and Buko, V. (2003) Dynamics of structural changes in rat liver after single dose of gamma-irradiation. Rad. Biol. Radioecol., 43(6): 618. 